\title{
Schutz ökologisch sensibler Fließgewässer: Konzepte und Fallbeispiele
}

\author{
Sigrid Scheikl · Carina Seliger · Andreas Loach · Sabine Preis · Rafaela Schinegger · Christoph Walder • \\ Stefan Schmutz · Susanne Muhar
}

Online publiziert: 12 . Juli 2016

(c) Der/die Autor(en) 2016. Dieser Artikel ist eine Open-Access-Publikation.

\begin{abstract}
Zusammenfassung Europäische Fließgewässer stehen unter starkem Nutzungsdruck. Dabei zählen Eingriffe in die Hydromorphologie im Rahmen energiewirtschaftlicher Nutzung oder durch Hochwasserschutzmaßnahmen zu den stärksten Belastungen. Heute gibt es nur noch wenige verbleibende natürliche oder naturnahe Gewässerstrecken. In Österreich befinden sich derzeit nur $15 \%$ der Wasserkörper in einem „sehr guten“ und $22 \%$ in einem „guten“ ökologischen Zustand. Um die Ziele der EU-Wasserrahmenrichtlinie zu erreichen, sind im Nationalen Gewässerbewirtschaftungsplan

nien und ausgewählte methodische Ansätze zusammengefasst. Anhand von drei methodisch aufeinander aufbauenden Fallbeispielen wird außerdem detailliert vorgestellt, wie Schutzpriorisierungen von Gewässerstrecken als Pre-planning-Instrumente herangezogen werden können. Außerdem wird eine Methodik vorgestellt, die bei bereits bestehenden Kraftwerksplanungen auf (über-)regionaler Ebene durch die Integration gewässerökologischer, naturschutzfachlicher und energiewirtschaftlicher Aspekte einen wesentlichen Beitrag bei der Entscheidungsfindung leisten kann.
\end{abstract} Maßnahmen definiert, mittels derer der gute Zustand der Wasserkörper erreicht werden soll bzw. künftige Beeinträchtigungen und Verschlechterungen des Zustandes verhindert werden sollen. Gleichzeitig gilt es, die Ziele der EU-Erneuerbare-Energien-Richtlinie $\mathrm{zu}$ erreichen. In Österreich spielt dabei insbesondere der weitere Ausbau der Wasserkraft eine wesentliche Rolle. Um sowohl Schutz- als auch Nutzungsziele zu erreichen bzw. um eine transparente Abwägung unterschiedlicher Interessen zu gewährleisten, ist eine strategische Planung notwendig. Im hier vorlegenden Artikel werden diesbezügliche Empfehlungen, Leitli-

DI S. S. Scheikl $(\bowtie) \cdot$ DDI C. C. Seliger .

DI A. A. Loach - DI S. S. Preis .

DI Dr. R. Schinegger .

ao.Univ.-Prof. DI Dr. S. Schmutz .

ao.Univ.-Prof. DI Dr. S. Muhar

Department für

Wasser-Atmosphäre-Umwelt, Institut für Hydrobiologie

und Gewässermanagement,

Universität für Bodenkultur Wien,

Max-Emanuel-Straße 17, 1180 Wien,

Österreich

E-Mail: sigrid.scheikl@boku.ac.at

Mag. C. Walder

WWF Österreich, Ottakringer Straße 114-116, 1160 Wien, Österreich
Schlüsselwörter Gewässerschutz • Strategische Planung .

Schutzkonzepte ·

Planungsinstrumente $\cdot$ DecisionSupport-Systeme

Protecting ecologically sensitive river stretches: concepts and case studies

Abstract European rivers are subject to heavy demand. In this regard, hydromorphological alterations in connection with energy production or flood protection measures are among the most serious issues. Today only very few natural or near-natural river reaches remain, and in Austria only 15\% of the rivers are classified as being in "very good" and a further $22 \%$ percent as being in "good" ecological condition. In keeping with the objectives of the EU Water Framework Directive, the defines measures intended to achieve a good ecological status and/or avoid the further deterioration of Austria's waterbodies.

At the same time, meeting the criteria of the EU Renewable Energy Directive is another important goal. In Austria, this especially involves the further expansion of hydropower. RecPriorisierungsansätze · Wasserkraft . National River Basin Management Plan onciling resource conservation with usage demands, and transparently balancing these competing interests, calls for strategic planning. The following paper provides an overview of recommendations, guidelines and selected methodological approaches. Further, it examines three case studies that build upon one another methodologically to demonstrate in detail how protection prioritization systems for river reaches can be employed as effective pre-planning instruments. Lastly, it presents a method by means of which hydroecological, nature conservation and energy production aspects can be integrated into already available power plant planning schemes at the (supra-) regional level to significantly facilitate decision-making processes.

Keywords Water conservation . Prioritization systems - Water power . Strategic planning - Protection concepts · Planning instruments . Decision support systems

\section{Einleitung und Hintergrund}

Mit Inkrafttreten der Wasserrahmenrichtlinie (WRRL; Richtlinie 2000/60/EG) im Jahr 2002 wurden die EU-Mitgliedstaaten verpflichtet, Maßnahmenprogramme zu entwickeln, durch die (u. a.) alle natürlichen Oberflächengewässer bis zum Jahr 2015 (längstens bis 2027) einen guten ökologischen Zustand erreichen. Schon im ersten Bericht der Europäischen Kommission über die Umsetzung der EU-Wasserrahmenrichtlinie wurde festgestellt, dass rund die Hälfte aller Wasserkörper dieses Qualitätsziel bis zum Jahr 2015 nicht erreichen würde. Als wesentlichste Hindernisse für die Zielerreichung wurden neben Übernutzung und Verschmutzung insbesondere hydromorphologische Belastungen genannt (Europäische Kommission 2012). Auch im folgenden Zwischenbericht über die Maßnahmenprogramme der Mitgliedstaaten 
wurde hervorgehoben, dass Eingriffe in das Abflussverhalten und in die physikalische Struktur von Wasserkörpern mitunter die Hauptgründe für die Zielverfehlung darstellen (Europäische Kommission 2015).

Diese Aussagen auf EU-weiter Ebene spiegeln sich auch in den Ergebnissen der Analysen zu den Nationalen Gewässerbewirtschaftungsplänen (NGPs) in Österreich wider. Die Belastungs- und Risikoanalyse für den ersten NGP 2009 ergab, dass rund $50 \%$ der Wasserkörper das Ziel des guten Zustandes innerhalb der ersten Planungsperiode (bis 2015) nicht erreichen würden. Als Ursache galten fast ausschließlich (zu $96 \%$ ) hydromorphologische Belastungen, die einerseits aus einer intensiven energiewirtschaftlichen Nutzung, in Form von Restwasser, Stau, Schwall, und auch Querbauwerken, andererseits aus umfassenden strukturellen Eingriffen, v. a. bedingt durch Hochwasserschutzmaßnahmen, resultieren (BMLFUW 2010). Nach der ersten 6-jährigen Planungsperiode hat sich das sichere Risiko der Zielverfehlung bis 2021 auf 38,3 \% verringert - bei weiterhin gleichbleibendem Anteil der hydromorphologischen Belastungen. Es wird im Entwurf zum NGP 2015 jedoch bereits darauf hingewiesen, dass es $u$. a. aus technischen und finanziellen Gründen nicht möglich sein wird, den guten Zustand bis $2027 \mathrm{zu}$ erreichen und entsprechende Maßnahmen auch über diese Frist hinaus gesetzt werden müssen (BMLFUW 2015).

Parallel zu diesen Entwicklungen und Vorgaben zielt die ErneuerbareEnergien-Richtlinie der EU (EE-RL, Richtlinie 2009/28/EG) darauf ab, bis zum Jahr 2020 den Energieverbrauch zu $20 \%$ aus erneuerbaren Energiequellen $\mathrm{zu}$ decken. Darauf basierend ist in der österreichischen Energiestrategie festgelegt, den Anteil erneuerbarer Energieträger bis 2020 von derzeit $33 \%$ auf $34 \% \mathrm{zu}$ erhöhen. Als Teilziel im Bereich der Stromerzeugung wurde der Ausbau der Wasserkraft bis zum Jahr 2015 um weitere 12,6 PJ (3,5 TWh) festgelegt (BMWFJ und BMLFUW 2010). So wurden zwischen 2009 und 2014 mindestens 97 neue Wasserkraftwerke gebaut bzw. befanden sich in diesem Zeitraum in Bau oder Ausbau (WWF Österreich 2014; Seliger et al. 2015). Im Jahr 2014 gab es in Österreich insgesamt 2.923 Wasserkraftwerke, die mit einer Leistung von 13.568 MW und einem jährlichen Gesamtregelarbeitsvermö- gen von $44.728 \mathrm{GWh} / \mathrm{a}$ einen Anteil von $68,7 \%$ an der gesamten Erzeugung elektrischer Energie in Österreich hatten (statistisch erfasste Kraftwerke, Energie-Control Austria 2015a, 2015b) ${ }^{1}$. Mit diesem Wert liegt Österreich weit über dem EU-Durchschnitt: Im Jahr 2012 lag der Anteil an Stromerzeugung aus Wasserkraft EU-weit bei rund $10 \%$, da insbesondere nicht erneuerbare Ressourcen (Gas, Öl, Kohle; 45 \%) und Nuklearenergie (27\%) den wesentlichen Anteil an der Stromerzeugung ausmachten (OECD und IEA 2014).

Nach Seliger et al. (2015) waren im Jahr 2013 jedenfalls 102 Wasserkraftwerke in Planung (Aus- und Neubauten). Eine Analyse dieser Projekte ergab, dass fast die Hälfte davon auch Wasserkörper betraf, die noch einen sehr guten (18 Projekte) oder guten (29 Projekte) ökologischen Zustand aufwiesen $(\mathrm{Mu}-$ har et al. 2013).

Vor dem Hintergrund des bereits hohen Ausbaugrades (68 \%; PÖYRY 2008) und des geplanten weiteren Ausbaues der Wasserkraft einerseits und der bereits hohen Belastung der österreichischen Fließgewässer durch Eingriffe in die Hydromorphologie stieg in den letzten Jahren auch das Bewusstsein für die Notwendigkeit einer strategischen Planung. Auch im Entwurf zum NGP 2015 wird betont, dass dem Erhalt der letzten unbeeinträchtigten Strecken „im Sinne einer nachhaltigen Bewirtschaftung" eine hohe Bedeutung zukommt (BMLFUW 2015). Die EU-Wasserdirektoren empfahlen für den zukünftigen Ausbau der Wasserkraft eine strategische Herangehensweise und die Ausweisung von aus ökologischer Sicht geeigneten bzw. nicht geeigneten Strecken („no-go areas" lt. CIS 2006).

Für den Alpenraum erstellte die Alpenkonvention einen Leitfaden für die strategische Bewertung neuer Kleinwasserkraftwerke. Hier wird empfohlen, in einem ersten Schritt auf regionaler Ebene eine Priorisierung von Gewässerstrecken hinsichtlich ihrer Eignung für Kleinwasserkraftwerke vorzunehmen. Die vorgeschlagenen Kriterien adressieren einerseits das Wasserkraftpotenzial, andererseits ökologische und landschaftliche Werte. Die Bewertung der Strecken erfolgt dabei in vier Stu-

${ }^{1}$ Ergänzt man diese Zahl um kleine Eigenbedarfsanlagen, so existieren derzeit rund 5.500 Wasserkraftwerke in Österreich (vgl. Habersack et al. 2011; Wagner et al. 2015]. fen von „gut geeignet“ bis „Ausschluss“ (Plattform Wasserwirtschaft im Alpenraum 2011).

Auch die Internationale Kommission zum Schutz der Donau empfiehlt eine strategische Herangehensweise bei der Planung neuer Wasserkraftwerke. Dabei sind „ökologische, soziale und wirtschaftliche Faktoren zu berücksichtigen“ (ICPDR 2013). Der vorgeschlagene Bewertungsansatz entspricht weitestgehend jenem der Plattform Wasserwirtschaft im Alpenraum. Beide Leitlinien schlagen auch mögliche Kriterien für die regionale/nationale und die standort- bzw. projektspezifische Bewertung vor. Eine Gewichtung bzw. Einzelwertung der Kriterien wird hingegen in den Leitlinien nicht vorgegeben.

In Österreich wurde im Jahr 2012 vom BMLFUW der „Österreichische Wasserkatalog“ erlassen (ÖWK; BMLFUW 2012). Er hat die Förderung einer nachhaltigen Wasserkraftnutzung zum Ziel und gibt Kriterien und Indikatoren zur Bewertung energiewirtschaftlicher, ökologischer und sonstiger wasserwirtschaftlicher Aspekte vor. „Als unverbindliche Richtschnur" soll er „Hilfestellung bei der Handhabung der einschlägigen wasserrechtlichen $\mathrm{Be}$ stimmungen bieten" (BMLFUW 2012) Als derzeit einziges Instrument in Österreich zielt er auf eine bundesweit einheitliche Herangehensweise bei der Abwägung energiewirtschaftlicher und ökologischer Interessen ab und stützt damit großmaßstäbliche strategische Planungen auf nationaler Ebene. Methodische Vorgaben für eine integrative Gesamtbewertung gibt es im ÖWK jedoch nicht.

Auf Ebene der Bundesländer bestehen seit mehreren Jahren verschiedenste Strategien, Gewässerstrecken zu definieren, die - je nach Priorisierungsansatz - gegenüber einem weiteren Ausbau der Wasserkraft besonders sensibel sind und/oder frei gehalten werden sollen. In Vorarlberg wurde z. B. im Zuge der Maßnahmenplanung bzgl. der Energieentwicklung festgelegt, dass bis 2030 Kleinwasserkraftprojekte außerhalb von Strecken mit einem sehr guten ökologischen Zustand als vorrangig angesehen werden (Amt der Vorarlberger Landesregierung 2011). Das Land Tirol verabschiedete per Regierungsbeschluss einen Kriterienkatalog „für die weitere Nutzung der Wasserkraft in Tirol“. Er soll mittels einer umfassenden Auswahl an Kriterien und Bewertungsmethoden $u$. a. eine transparente 
„Abwägung der technischen, ökonomischen, ökologischen und gesellschaftlichen Interessen" ermöglichen (Amt der Tiroler Landesregierung 2011). Im Bereich der Gewässerökologie und des Naturschutzes gibt er eine Methodik vor, die Sensibilität von Gewässerstrecken in 3 Stufen zu beurteilen. Außerdem werden Ausschluss-Strecken von Rechts wegen definiert (z. B. TNSchG 2005, Nationalparkgesetz). Gleicherweise zielen die Regionalprogramme in Niederösterreich ${ }^{2}$ und der Steiermark („Gewässerschutzverordnung“, LGBl. Nr. 40/2015) auf eine strategische Abwägung energiewirtschaftlicher und ökologischer/landschaftlicher Aspekte bei der Planung eines weiteren Ausbaues der Wasserkraft ab. In Oberösterreich wurde im Rahmen einer „Wasserkraftpotenzialanalyse“ die Sensibilität von Gewässertsrecken bewertet um in $\mathrm{Zu}$ sammenschau mit einer energiewirtschaftlichen Analyse ein „ökologisch verträgliches und nachhaltig nutzbares Ausbaupotential“ zu ermitteln (Amt der Oberösterreichischen Landesregierung 2015).

Im Folgenden werden drei methodisch aufeinander aufbauende Fallbeispiele vorgestellt, in deren Rahmen sierung strategischer nationaler und regionaler Strategien - bundesweite Priorisierungen der Schutzwürdigkeit von Gewässerstrecken durchgeführt wurden. Es werden sektorale naturschutzfachlich-ökologische Beurteilungsmethoden vorgestellt und verglichen sowie Ansätze zur Harmonisierung von energiewirtschaftlichen und ökologischen Ansprüchen vorgestellt. Die ausgewählten Fallbeispiele und die dort angewandten Methoden können sowohl im Vorfeld von Planungen im Sinne eines strategischen Pre-Plannings, als auch bei schon bestehenden Planungen als Entscheidungshilfe herangezogen werliegen Studien und zugehörige Einzelpublikationen und Berichte zugrunde, an denen die AutorInnen des vorliegenden Artikels beteiligt waren. im Sinne der Forcierung und Harmoniden. Den ausgewählten Fallbeispielen

\section{Fallbeispiele}

2.1 Fallbeispiel 1: Priorisierung der Schutzwürdigkeit von

Fließgewässern auf nationaler Ebene

Eine systematische Einstufung der Schutzwürdigkeit aller österreichischen Fließgewässer mit einem Einzugsgebiet $(\mathrm{EZG}) \geq 10 \mathrm{~km}^{2}$, also des gesamten österreichischen Berichtsgewässernetzes (BMLFUW), erfolgte erstmals im Jahre 2010 durch das Institut für Hydrobiologie und Gewässermanagement (IHG) der Universität für Bodenkultur Wien (BOKU). Die Ausweisungen erfolgten nach naturschutzfachlichen und gewässerökologischen Gesichtspunkten im Rahmen der fachlichen Analysen zur WWF-Initiative „Ökomasterplan Stufe II" (ÖMP II; WWF Österreich 2010). Primäres Ziel der Studie war die Entwicklung fachlicher Grundlagen, die die Entscheidungsfindung für eine strategische Planung hinsichtlich des Wasserkraftausbaus in Österreich unterstützen sollten.

Mit den Daten des ersten Nationalen Gewässerbewirtschaftungsplans 2009 (NGP 2009; BMLFUW 2010) standen erstmalig flächendeckende, konsistent erhobene und klassifizierte Informationen zu Zustand und Belastungen des österreichischen Fließgewässernetzes (EZG $\geq 10 \mathrm{~km}^{2}$ ) zur Verfügung. Diese bildeten zusammen mit naturschutzfachlichen Informationen zu Schutzgebietsausweisungen die Basis für den Priorisierungsprozess. Insgesamt vier sektorale Kriterien wurden in einem ersten Schritt entweder nach bereits vorhandenen Einstufungen des NGP oder nach darüber hinausgehenden fachlichen Kriterien weiter differenziert, um sie weiter in die Schutzwürdigkeitsklassen „sehr hoch“, „bedingt“ und "gering schutzwürdig“ einzuteilen ${ }^{3,4}$ :

1. Ökologischer Zustand der Wasserkörper. Differenzierung nach der fünfstufigen Bewertung gemäß WRRL unter Berücksichtigung der im NGP angeführten „Sicherheit der Bewertung“. Wasserkörper ohne Bewertung des ökologischen Zustands

3 Strecken mit Stau- oder Restwasserbelastung wurden von der Bewertung ausgenommen.

2 Die Verordnung „NÖ wasserwirtschaftliches Regionalprogramm 2016 zum Erhalt von wertvollen Gewässerstrecken“ ist derzeit (April 2016) in Vorbereitung.
${ }^{4}$ Für eine detaillierte Beschreibung der Kriterien siehe WWF Österreich (2010). wurden bei diesem sektoralen Kriterium nicht berücksichtigt.

2. Lage von Fließgewässern oder Fließgewässerabschnitten in Schutzgebieten: Es wurden nationale, internationale und EU-spezifische Schutzgebietskategorien berücksichtigt, wobei keine weitere Differenzierung erfolgte.

3. Hydromorphologischer Zustand der Wasserkörper. Differenzierung nach der fünfstufigen Bewertung gemäß WRRL. Wasserkörper ohne Bewertung des ökologischen Zustands wurden in diesem sektoralen Kriterium nicht berücksichtigt.

4. Länge der zusammenhängenden freien Fließstrecke: Differenzierung der Länge der Fließstrecke (ohne Stau oder Restwasser) zwischen zwei Kontinuumsunterbrechungen (nicht passierbare Querbauwerke, Kraftwerke) in Abhängigkeit von der Fischregion.

Darauf aufbauend erfolgte die Priorisierung auf zwei Ebenen: In der ersten Ebene wurden die Schutzwürdigkeitsklassen so gewichtet, dass das jeweils am höchsten klassifizierte Kriterium am Gewässer für die weitere Bewertung ausschlaggebend war. In der zweiten Ebene wurden die sektoralen Kriterien gewichtet und entsprechend ihrer Klassifizierung nach Sensitivitätsklassen priorisiert (Abb. 1).

Insgesamt zeigte sich, dass noch rund $47,5 \%$ aller untersuchten Fließgewässer als „sehr hoch schutzwürdig“ einzustufen waren $(33,4 \%$ aufgrund des ökologischen Zustands und 14,1 \% aufgrund ihrer Lage in Schutzgebieten) (Abb. 2; vgl. WWF Österreich 2010). Insbesondere für diese Strecken wurde im Rahmen des ÖMP II empfohlen, den natürlichen/naturnahen Zustand $\mathrm{zu}$ erhalten bzw. ihren aktuellen Schutzstatus sicherzustellen. Hinsichtlich der energiewirtschaftlichen Nutzung wurde für diese Strecken auch die Ausweisung von „no-go Areas“ empfohlen.

Als „hoch schutzwürdig“ aufgrund des hydromorphologischen Zustands bzw. der Länge der freien Fließstrecke wurden weitere $8,2 \%$ der Gewässerstrecken beurteilt. Für diese Strecken wurde insbesondere aufgrund ihres hohen Sanierungs- bzw. Revitalisierungspotenzials die Notwendigkeit ihrer Erhaltung hervorgehoben. Die als ,gering schutzwürdig“ und "bedingt schutzwürdig“ beurteilten Strecken wurden ohne einer Detailbeurteilung im Rah- 


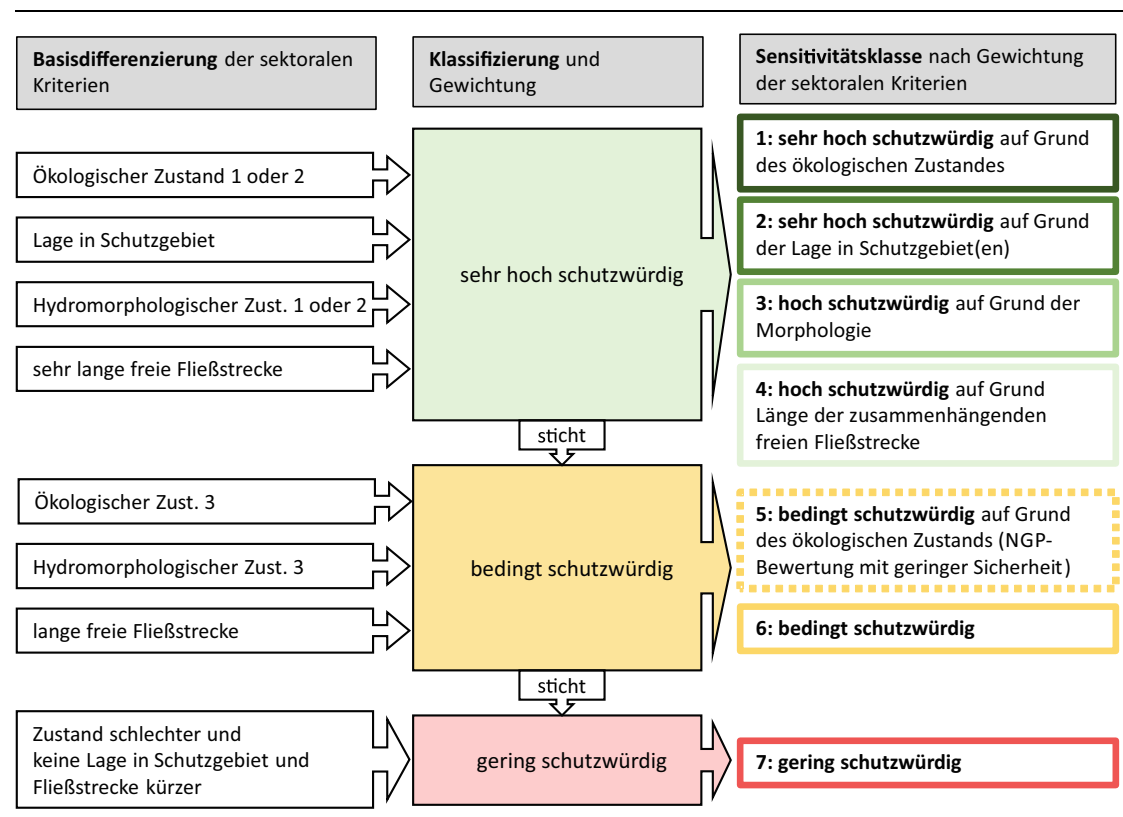

Abb. 1 Prozess der Schutzpriorisierung von Fließgewässern im Rahmen des ÖMP II (Nach WWF Österreich 2010)

men der vorgeschriebenen Verfahren zur Projektbewilligung vorzugreifen grundsätzlich als potenzielle Strecken für die nachhaltige Wasserkraftnutzung klassifiziert.

Ausgehend von den Ergebnissen dieser Studie sowie dem Vorliegen verschiedenster Kriterienkataloge und Leitfäden für die Beurteilung des Wasserkraftausbaus bzw. der Fließgewässersensibilität auf Bundes- und Länderebene (s. oben), wurde im Jahr 2014 der Ökomasterplan Stufe III (ÖMP III) erarbeitet (WWF Österreich 2014; siehe auch Fallbeispiel 2: „Hy:Con“). Im Rahmen der dem ÖMP III zugrunde liegenden fachlichen Studie „Hy:Con“ wurden die ökologischen Kriterien umfassend erweitert und die Sensitivitätsklassen um die Klasse „Ausschluss - bezogen auf den Wasserkraftausbau“ ergänzt. Die Endbewertung erfolgte unter Berücksichtigung verschiedener Schutzszenarien (s. unten). Dabei wurden zwar ausschließlich von Kraftwerksprojekten betroffene Strecken bewertet, jedoch wurden im Zuge der Vorarbeiten die Ausschluss-Kriterien am gesamten österreichischen Gewässernetz $\left(E Z G \geq 10 \mathrm{~km}^{2}\right.$ ) analysiert. Eines dieser Szenarien (,Szenario 2: WWF Energiewende“) wird im Folgenden mit den Ergebnissen des ÖMP II verglichen und diskutiert:

Wurde im ÖMP II noch für alle Schutzgebietskategorien eine Ausweisung als „No-Go Area“ empfohlen, so wurden diese nun im Szenario „WWF Energiewende" nach ihrer rechtlichen levanz differenziert beurteilt. Der sehr gute ökologische Zustand wurde zu einem Ausschlusskriterium und der gute ökologische Zustand als „sehr hoch“ eingestuft. Das Kriterium „Hydromorphologie" wurde neu definiert und von Wasserkörpern auf die Ebene der NGPBelastungsanalyse (500m-Abschnitte der Morphologiebewertung) heruntergebrochen. Morphologisch nicht veränderte Strecken ab einer Länge > 1 Kilometer wurden nun ebenfalls in den Ausschluss aufgenommen. Als neue Ausschlusskriterien kamen außerdem Auenflächen mit überragender naturschutzfachlicher Bedeutung (nach Lazowski et al. 2011) und Flussheiligtümer (nach BMFL et al. 1998) hinzu.

Im Szenario „WWF Energiewende“ wurden nun rund $39 \%$ des österreichischen Gewässernetzes als Ausschluss (bezogen auf den Ausbau der Wasserkraft) eingestuft (Abb. 3). Auffallend ist hier, dass gerade die beiden Kriterien, die im ÖMP II die sehr hohen Sensitivitätsklassen bildeten, in der Studie zum ÖMP III in ihrer Bedeutung jeweils um mehr als die Hälfte reduziert wurden. Dies ist dadurch zu begründen, dass nur noch der sehr gute ökologische Zustand den Ausschluss bedeutete und Schutzgebiete nun nach ihrer Relevanz für den Fließgewässerschutz differenziert betrachtet wurden und da„Schutzstärke“ bzw. ihrer Gewässerre-

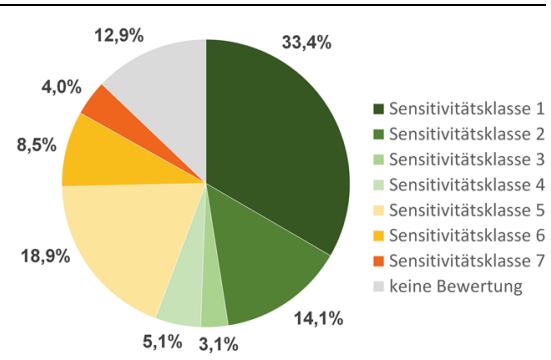

Abb. 2 Schutzwürdigkeit der österreichischen Fließgewässer nach Sensitivitätsklassen. Ergebnisse der Analysen im Rahmen des ÖMP II. (WWF Österreich 2010)

mit nicht mehr grundsätzlich zum Ausschluss führten. Die höchste Bedeutung bei der Ausweisung von AusschlussStrecken kam nun dem morphologischen Zustand zu: Rund zwei Drittel aller Ausschluss-Strecken (und damit rund $29 \%$ des untersuchten Gewässernetzes) setzten sich aus Strecken zusammen, die laut den Daten des NGP 2009 als morphologisch unverändert galten. An nur rund 3,5 \% aller Ausschluss-Strecken befanden sich noch Auenflächen mit „überragender naturschutzfachlicher Bedeutung“ (nach Lazowski et al. 2011) - eines der Kriterien, die in Hy:Con ergänzt wurden.

Im direkten Vergleich der beiden Ansätze (siehe Abb. 4) zeigte sich, dass die Ergebnisse in weiten Teilen übereinstimmten (insgesamt rund $8.000 \mathrm{~km}$ waren gleichermaßen als „sehr hoch schutzwürdig“ und als „Ausschluss“ ausgewiesen). Im Rahmen des ÖMP III wurden vorwiegend in Oberösterreich, Niederösterreich und der südöstlichen Steiermark über den ÖMP II hinausgehende Strecken von insgesamt rund $1.800 \mathrm{~km}$ ausgewiesen. Auf ca. $80 \%$ dieser Strecken war der morphologische Zustand zumindest eines der ausschlaggebenden Kriterien.

Hingegen wurden ca. $5.100 \mathrm{~km}$ ausschließlich im ÖMP II als sehr hoch schutzwürdig ausgewiesen. Diese Strecken bestanden zu $56 \%$ aus Wasserkörpern mit einem guten ökologischen Zustand. In 44 \% dieser Fälle waren ausschließlich Schutzgebiete ausschlaggebend, deren Relevanz für den Gewässerschutz im Rahmen des ÖMP III niedriger eingestuft wurde. Entweder bezieht sich der Schutz bei diesen Kategorien nicht dezidiert auf "Gewässerschutz“ (wie es z. B. bei RAMSAR-Gebieten der Fall wäre) oder mit ihrem Schutzstatus geht aus rechtlicher Sicht nicht auto- 


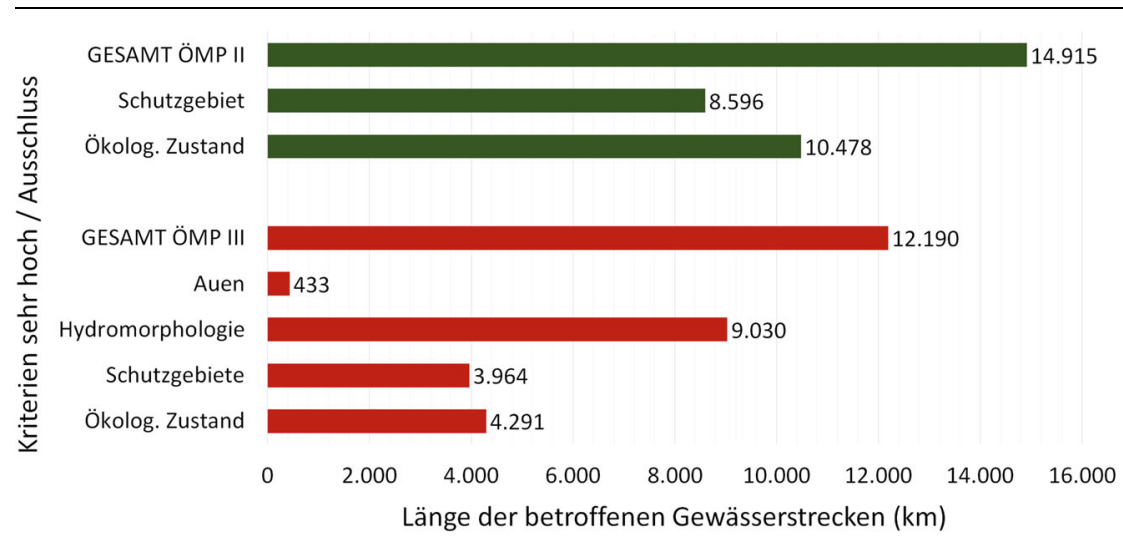

Abb. 3 Gesamtlänge der empfohlenen „No-Go Areas“ nach ÖMP II (Sensitivitätsklassen 1 und 2; grün) und der ausgewiesenen Ausschluss-Strecken nach Hy:Con/ÖMP III (rot) sowie Verteilung der sektoralen Kriterien

matisch auch ein Verbot von Eingriffen einher (im Gegensatz zu z. B. Nationalparks oder Sonderschutzgebieten).

\subsection{Fallbeispiel 2: Hy:Con - Ein} integratives Decision Support System zur Harmonisierung nationaler energiewirtschaftlicher und naturschutzfachlicher Ansprüche auf Projektebene

"Hydropower development and Conservation needs“ - kurz „Hy:Con“ - wurde im Zuge der Studie „Anwendung unterschiedlicher ökologischer und energiewirtschaftlicher Kriterien zur integrativen Bewertung von Wasserkraftprojekten“ entwickelt und bildete die fachliche Grundlage für den Ökomasterplan III (WWF Österreich 2014; Seliger et al. 2015). Hy:Con beschreibt ein methodisch transparentes Bewertungssystem, das Entscheidungsträger bei einer strategischen Abwägung von naturschutzfachlichen bzw. gewässerökologischen und energiewirtschaftlichen Aspekten unterstützen soll.

Nachdem zuvor, im Rahmen der Studie zum ÖMP II, bereits die besonders schützenswerten Gewässerstrecken in Österreich nach ihrer Sensitivität priorisiert und ausgewiesen wurden (WWF Österreich 2010), wurden nun mittels aktualisierter Daten und Kriterien u. a. Strecken identifiziert, an denen aus ökologischen Gesichtspunkten kein weiterer Ausbau der Wasserkraft stattfinden sollte („Ausschluss-Strecken“, vgl. auch Fallbeispiel 1).

Primäres Ziel, und Neuerung gegenüber der Studie zum ÖMP II, war es jedoch, einen integrativen Ansatz zu entwickeln, der im Kontext teils nationaler und internationaler Gesetze,
Strategien und Richtlinien ${ }^{5}$ die Identifikation von Wasserkraftprojekten mit hoher energiewirtschaftlicher Attraktivität und geringem Konfliktpotenzial mit Naturschutz und Aspekten der Gewässerökologie erlauben sollte.

Das Hy:Con-Modell und die daraus resultierenden Ergebnisse ersetzen jedoch keine abschließende Einzelfallbeauch aus energiewirtschaftlicher Sicht nicht alle lokalen Gegebenheiten und genehmigungsrelevanten Parameter im Detail berücksichtigt werden konnten. Das Modell stellt jedoch einen großmaßstäblichen methodischen Ansatz für die Analyse des Ist-Zustandes dar und kann somit einen wesentlichen Beitrag zu strategischen und großmaßstäblichen energiepolitischen Entscheidungen leisten.

Die für die sektoralen Bewertungen herangezogenen gewässerökologischen und energiewirtschaftlichen Kriterien und Indikatoren basierten vorwiegend auf den Vorgaben des ÖWK (BMLFUW 2012). Um die Kriterien für Gewässerschutz möglichst umfassend abzudecken, wurden zusätzlich naturschutzfachliche Kriterien (Schutzgebiete im Sinne des Naturschutzes) aus der Studie zum ÖMP II übernommen und deren Gewichtung bzw. Bewertung aktualisiert. Der ÖWK wurde als Grundlage gewählt, weil er als Erlass des BM-

5 Insbesondere Wasserrahmenrichtlinie 2000/60/EG, Fauna-Flora-Habitat-Richtlinie 92/43/EWG und Erneuerbare-Energien-Richtlinie 2009/28/EG und damit verbunden auch Naturschutzgesetze der Länder, Wasserrechtsgesetz (WRG 1959) und Energiestrategien des Bundes und der Länder. wertung, da sowohl aus ökologischer als
LFUW aktuell als einziges legislatives Instrument auf Bundesebene eine einheitliche Betrachtung und Beurteilung von Wasserkraftprojekten vorsieht. Er gibt ausgewählte Kriterien sowie die grundlegende Bewertung ihrer zugehörigen Indikatoren vor. Ein methodischer Ansatz für eine integrative ökologischökonomische Beurteilung von Wasserkraftwerksprojekten wurde im Zuge von Hy:Con entwickelt.

\subsubsection{Datengrundlagen}

Kraftwerksprojekte (KWPs): Die Datenbank der Wasserkraftwerksprojekte (KWPs) bildete einen zentralen Bestandteil von Hy:Con. Sie beinhaltete bewertungsrelevante Informationen $\mathrm{zu}$ den einzelnen, im Zuge der vorangegangenen umfassenden Recherche erhobenen Neu- und Ausbauprojekten (insgesamt 102 KWPs).

Durch Lageinformationen konnten die Kraftwerke am Gewässernetz verortet werden. In weiterer Folge wurden deren räumlicher Wirkungsbereich (hinsichtlich Schwall, Ausleitung und Rückstau) definiert. War der Wirkungsbereich nicht bekannt, so wurde er basierend auf der EZG-Größe abgeschätzt. Die so definierten Gewässerabschnitte bildeten den räumlichen Bezug für die ökologische Bewertung, da je KWP nur jene Gewässerabschnitte in Hinblick auf ihr Konfliktpotenzial untersucht wurden, die sich im entsprechenden Einflussbereich befanden. Als wesentliche Parameter für die energiewirtschaftliche Bewertung beinhaltete die Datenbank für jedes KWP Informationen zu:

- Kraftwerkstyp: Laufkraftwerk mit oder ohne Stundenspeicher oder Ausleitung,

- Leistung (MW),

- Regelarbeitsvermögen (RAV in GWh/ a),

- Größenklasse (Kleinst: < 1 MW; Klein: 1 bis < 10 MW; Mittel: 10 bis < 20 MW; Groß: > $20 \mathrm{MW}$ ),

- ggf. Speicherkapazität ( $\mathrm{m}^{3}$ und GWh),

- ggf. Pumpleistung (MW),

- spezifische Investitionskosten $(€ / \mathrm{kWh})$.

Ökologische Kriterien: Bezüglich des Prüffeldes „Ökologische Kriterien“ gibt der ÖWK (BMLFUW 2012) insgesamt 4 relevante Kriterien (Natürlichkeit, Seltenheit, ökologische Schlüsselfunktion, räumliche Ausdehnung der negativen Wirkungen) vor, die in direktem Zusam- 


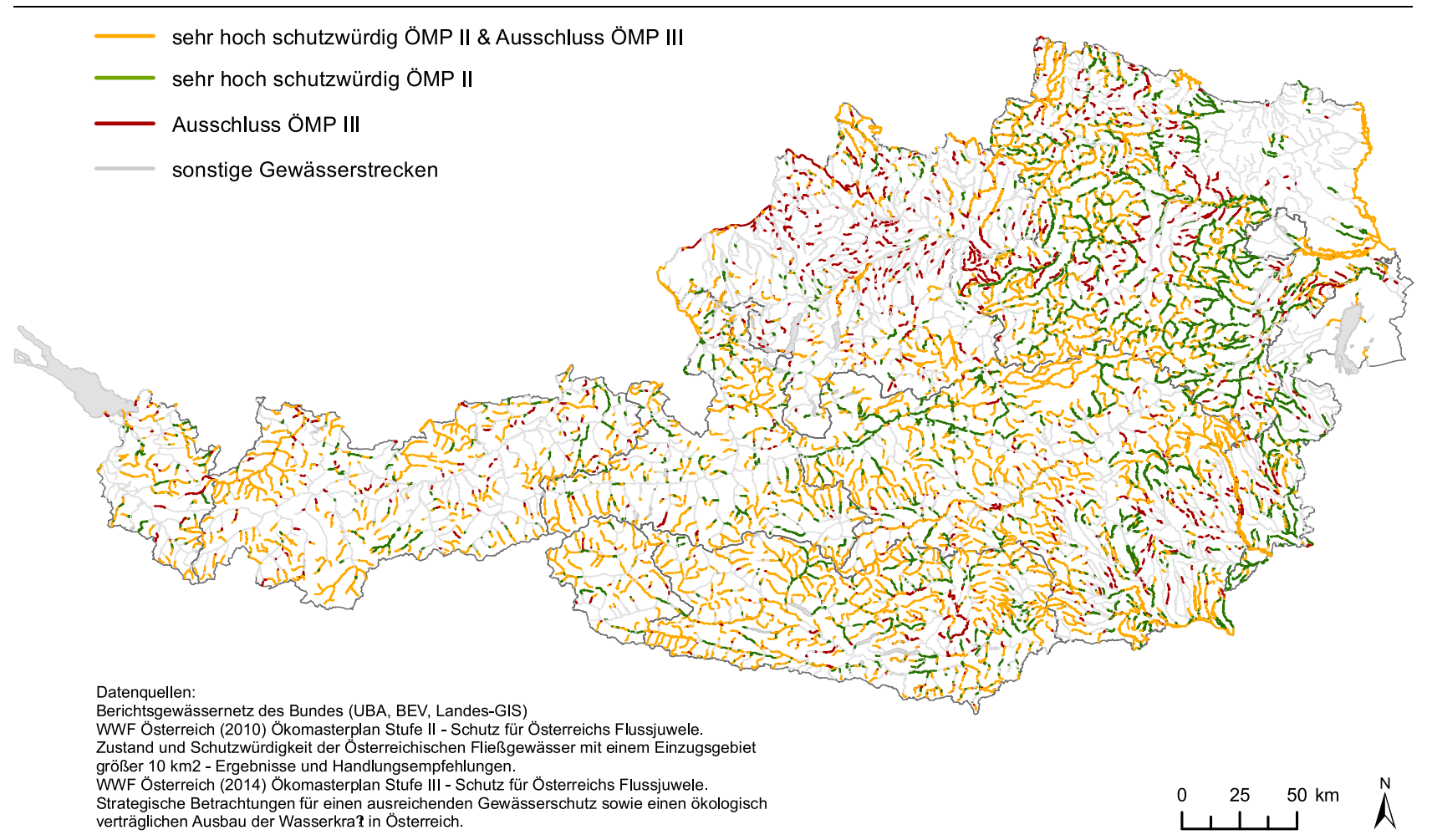

Abb. 4 Überlagerung der Ergebnisse: höchste Sensitivitätsklasse nach ÖMP II bzw. Ausschluss-Strecken nach ÖMP III

menhang mit hydromorphologischen Veränderungen im Rahmen der Wasserkraftnutzung stehen. Für jedes Kriterium wurden dabei mehrere quantifizierbare bzw. differenzierbare und damit direkt bewertbare Indikatoren identifiziert. Diese Indikatoren wurden neben den Kriterien des ÖMP II (WWF Österreich 2010) als Basis für die Auswahl und Definition bewertungsrelevanter ökologischer Kriterien im Rahmen von Hy:Con herangezogen. In einem mehrstufigen Prozess wurden schließlich von ursprünglich 50 potenziellen Einzelkriterien 39 Kriterien in das Bewertungsschema aufgenommen. Dabei mussten alle gewählten Kriterien mindestens folgende Ansprüche erfüllen: möglichst flächendeckende Datenverfügbarkeit bei konsistenter Erhebungsmethode, keine Redundanz zu anderen Kriterien, hohe Aussagekraft gegenüber hydromorphologischen Veränderungen und/ oder rechtliche Relevanz. Ein für die folgende Bewertung wesentlicher nächster Schritt war die Zuordnung der Einzel-

${ }^{6}$ Für eine detaillierte Beschreibung der Einzelkriterien und deren weitere Differenzierung siehe WWF Österreich (2014) und Seliger et al. (2015). kriterien zu jeweils einer von insgesamt 8 thematischen Gruppen (A bis $\mathrm{H})^{6}$ :

A. Ökologischer Zustand.

B. Hydromorphologischer Zustand: definiert durch den morphologischen Zustand auf standardisierten 500$\mathrm{m}$-Abschnitten sowie durch die Beeinflussung durch Restwasser, Stau und/oder Wanderhindernisse.

C. Schlüsselhabitate: mit den 9 Kriterien „Seltenheit Allgemeine Fließgewässertypen“, „Gletscherbach“, „Großer Fluss“, „Seeausrinn“, „Тypspezifische Ausprägungen“ (u. a. Klammen, Wasserfälle, Kaskaden, Schluchten), „Zubringer im Epirhithral“, „Zubringer Metarhithral“ (umfasst auch Hyporhithral klein und Epipotamal klein), „Zubringer Laichhabitate" (Zubringer zu Fließgewässern mit einem EZG $>500 \mathrm{~m}^{2}$ ) und „Laichhabitate Seeforelle“ (aktuell und potenziell).

D. Schlüsselarten: mit den 11 Kriterien Karausche (Carassius carassius), Renke (Coregonus sp.), Moderlieschen (Leucaspius delineates), Nerfling (Leuciscus idus), Wolgazander (Sander volgensis), Huchen (Hucho hucho), Äsche (Thymallus thymallus), Nase (Chondrostoma nasus), Dohlenkrebs (Austropotamobius pal- lipes), Flussperlmuschel (Margaritifera margaritifera), Deutsche Tamariske (Myricaria germanica).

E. Bewertung Auen (nach Lazowski et al. 2011).

F. Rechtlich gesicherte Schutzgebiete: mit den 3 Kriterien Nationalpark, Sonderschutzgebiet, Wildnisgebiet.

G. Sonstige Schutzgebiete: mit den 9 Kriterien Flussheiligtum (gemäß BMFL et al. 1998), Landschaftsschutzgebiet, Naturdenkmal, Naturschutzgebiet, Ruhegebiet, Natura-2000Gebiet, RAMSAR-Gebiet, WWRL-relevantes Natura 2000 Gebiet, anderes Schutzgebiet.

H. Freie Fließstrecke/Migrationskorridor: mit den 4 Kriterien Freie Fließstrecke (gemäß der Definition des ÖMP II), Seltenheit Freie Fließstrecke kleine Fließgewässer, Seltenheit Freie Fließstrecke mittlere und große Fließgewässer sowie Migrationskorridor.

\subsubsection{Das Bewertungsmodell}

Das Hy:Con-Modell setzt sich aus einer stufenweisen Datenbearbeitung und -bewertung zusammen, die schlussendlich zu einer integrativen ökologisch-ökonomischen Gesamtbeurteilung von Wasserkraftwerksprojekten 


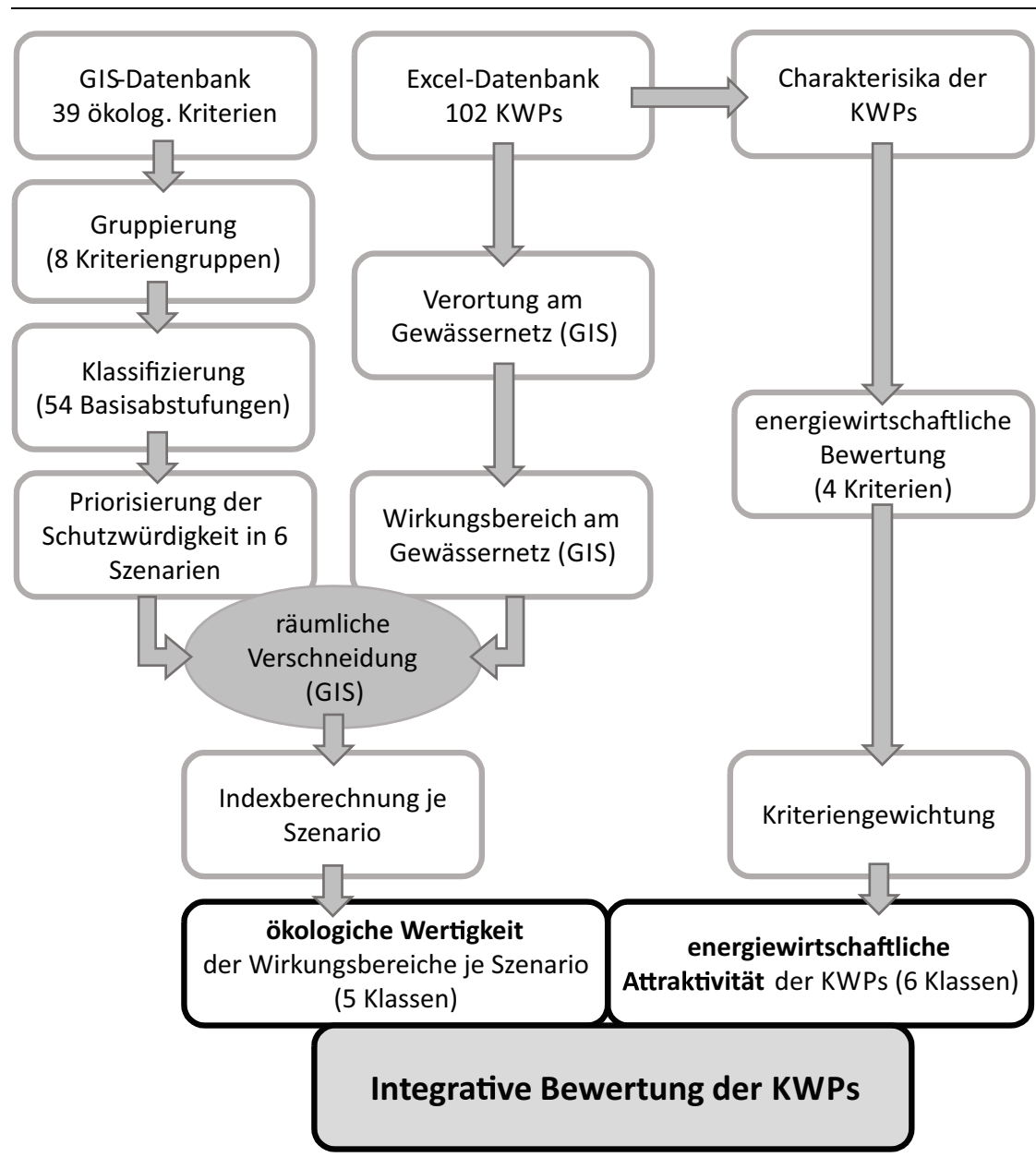

Abb. 5 Modell des integrativen Hy:Con-Bewertungsprozesses

führt (Abb. 5).Zunächst wurden basierend auf den identifizierten Kriterien separierte ökologische und energiewirtschaftliche Bewertungen durchgeführt. Die ökologische Bewertung der Kraftwerksprojekte erfolgte dabei szenarienbasiert: Um potenzielle Trends in Bezug auf die Gewichtung naturschutzfachlicher und gewässerökologischer Aspekte berücksichtigen zu können, wurden vier ökologische „Schutzszenarien“ entwickelt. Dabei wurden die Einzelkriterien und deren Basiseinstufung im Wirkungsbereich der KWPs je nach Szenario bezüglich derer ökologischen Wertigkeit und damit auch Konfliktpotenzial unterschiedlich hoch bzw. niedrig gewertet (in den fünf Klassen gering, mittel, hoch, sehr hoch, Ausschluss). Ergänzend wurden ÖWKund ÖMP-II-Szenarien entwickelt und berechnet, die hier nicht weiter erläutert werden (für Beschreibungen dieser Szenarien s. Seliger et al. 2015).

Die Bewertung der energiewirtschaftlichen Attraktivität erfolgte unab- hängig von den Szenarien auf Basis der generellen Charakteristika der KWPs. Für jedes KWP wurden zunächst die vier Kriterien „Wirtschaftlichkeit“, „Versorgungssicherheit“, „Versorgungsqualität“ und „Klimaschutz" einzeln bewertet. Anschließend wurden die Kriterien unterschiedlich gewichtet (Kriterien, die dieselben primären Eingangsparameter hatten, wurden niedriger gewichtet) und zu einer Gesamtbewertung der energiewirtschaftlichen Attraktivität zusammengeführt. Die Bewertung erfolgte mittels Vergabe von 6 Punkten (von "gering“ = 0 bis „hoch“ $=5)^{7}$.

Schlussendlich wurden die beiden sektoralen Ergebnisse überlagert, um darzustellen, welche KWPs aus energiewirtschaftlicher Perspektive als besonders attraktiv einzustufen sind und

7 Methodische Details und Ergebnisse s. WWF Österreich (2014) und Seliger et al. (2015). gleichzeitig nur geringes ökologisches Konfliktpotenzial aufweisen.

\subsection{3 Ökologische Bewertung}

Nachdem die 39 ökologischen Kriterien $\mathrm{zu}$ den acht oben beschriebenen thematischen Gruppen aggregiert wurden, erfolgte in einem weiteren Schritt für jedes Einzelkriterium eine Basisabstufung. Dafür wurden entweder bereits vorhandene Abstufungen verwendet (z. B. ökologischer Zustand, naturschutzfachliche Bedeutung von Auenflächen oder eine bereits erfolgte Einstufung in vorhandenen Datensätzen zum ÖWK), oder sie wurden unter Berücksichtigung fachlich-ökologischer Gesichtspunkte definiert (z. B. Differenzierung nach Biomasse bei Fischartenvorkommen). Waren in den Datensätzen keine Parameter vorhanden, die eine Differenzierung ermöglichten, so wurde das Vorkommen oder die Abwesenheit des Kriteriums auf der untersuchten Gewässerstrecke gewertet. Insgesamt standen damit als Sub-Kriterien 54 Basisabstufungen für die Bewertung zu Verfügung (s. auch Abb. 7).

Diese Abstufungen waren Ausgangspunkt für die Beurteilung des ökologischen Konfliktpotenzials in den verschiedenen Szenarien: Die Wertigkeit ökologischer Kriterien nimmt von Szenario 1 bis 4 kontinuierlich ab. Naturgemäß findet sich in den Szenarien 1 (SZ 1: Strenger Schutz) und 2 (SZ 2: WWF-Energiewende) eine vergleichsweise hohe Anzahl an Ausschluss- und sehr hoch bewerteten Kriterien. Parallel dazu steigt die Anzahl an hoch, mittel und geringen Wertungen hin $\mathrm{zu}$ SZ 4: Minimaler Schutz (Abb. 6).

Um zu einer ökologischen Gesamtbewertung der Gewässerstrecken im Einflussbereich der KWPs zu gelangen, konnte aufgrund der hohen Anzahl an Einzelkriterien die Methodik des ÖMP II nicht mehr angewendet werden. Für die Bewertung wurde ein Index entwickelt, der - im Gegensatz zum Stichprinzip des ÖMP II alle Kriterien gleichermaßen berücksichtigte und schrittweise $\mathrm{zu}$ einer Gesamtbeurteilung der Wirkungsgereiche je KWP und Szenario führte:

1. Zuweisung von Punkten für die ökologische Wertigkeit (Ausschluss $=4$; Sehr Hoch $=3$, Hoch $=2$, Mittel $=1$, Gering $=0$ ).

2. Automatisches Ausscheiden aller Kraftwerke, in deren Wirkungsbe- 


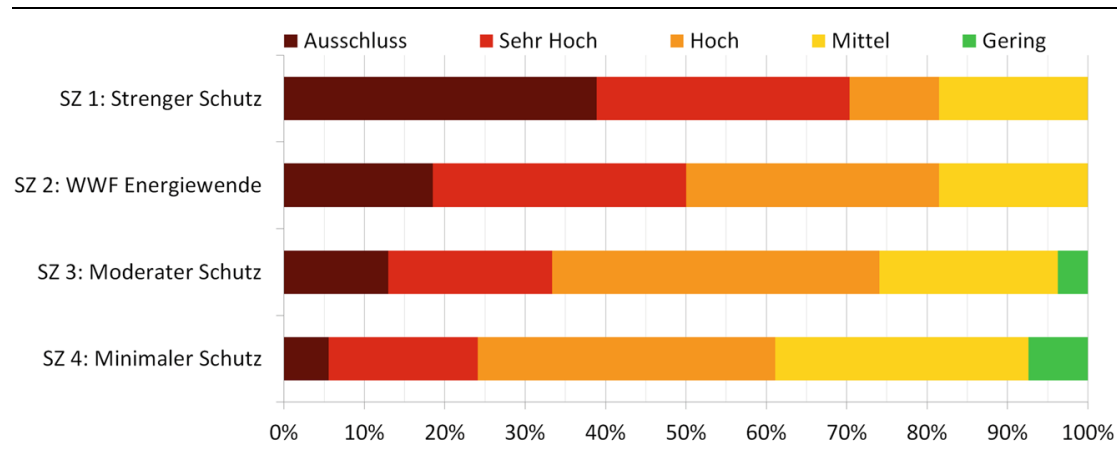

Abb. 6 Verteilung der „ökologischen Wertigkeiten“ der Kriterien auf die Szenarien

reich mindestens ein AusschlussKriterium lag.

3. Stichprinzip innerhalb der thematischen Gruppen: für jedes KWP wird je Gruppe jenes Kriterium mit der höchsten Bewertung für die weitere Berechnung ausgewählt. Diese Herangehensweise ist auch im Sinne des ÖWK, der vorgibt, dass sich die Gesamtbeurteilung der Kriterien aus der höchsten Beurteilung der Indikatoren (Hy:Con-Kriterien) ergibt. Dadurch ergeben sich acht Werte mit jeweils maximal 3 Punkten.

4. Mittelung der acht Gruppenwerte: Berechnung der arithmetischen Mittel je KWP.

5. Endbewertung der ökologischen Wertigkeit und damit des Konfliktpotenzials durch Definition gleicher Klassenbreiten basierend auf dem höchsten errechneten Mittelwert (über alle Szenarien hinweg war dies 2,7): 2,026-2,700 = Sehr hoch; 1,351-2,025 = Hoch; 0,676-1,350 = Mittel; 0,000-0,675 = Gering.

\subsubsection{Ergebnisse}

Verteilung der Subkriterien in den Wirkungsbereichen der KWPs: Nachdem die Basisabstufungen (im Sinne von Sub-Kriterien) mit den Wirkungsbereichen der KWPs verschnitten wurden, wurde festgestellt, dass nur 43 der insgesamt 54 Abstufungen auf von KWPs betroffenen Gewässertrecken lagen (vgl. Abb. 7). Von den 11 Subkriterien, die nicht in den Wirkungsbereichen lagen, waren drei als rechtlich gesicherte Schutzgebiete (Nationalpark, Sonderschutzgebiet und Wildnisgebiet) über alle Szenarien hinweg als Ausschlusskriterium bewertet. Die restlichen acht Subkriterien setzten sich ausschließlich aus dem Vorkommen bestimmter Schlüsselarten zusammen (Dohlen- krebs, Flussperlmuschel, Karausche, Moderlieschen, Renke, Wolgazander und Nase mit Biomasse $>20 \mathrm{~kg}$ ). Dabei handelt es sich ausschließlich um Arten, die entweder natürlich ein vergleichsweise kleines und abgegrenztes Verbreitungsgebiet in Österreich haben und/oder entsprechend Roter Listen (Reischütz und Reischütz 2007; Wolfram und Mikschi ; Petutschnig 2009) bzw. FFH-Richtlinie (92/43/EWG Anhang II) aufgrund ihrer hohen Gefährdung besonders schützenswert sind. Auch mögliche Datenlücken sind hier zu berücksichtigen.

Bei fast $50 \%$ aller KWPs ist das Subkriterium „Freie Fließstrecke“ für die Ausweisung als „sehr hoch schutzwürdig" mit ausschlaggebend. Damit betraf fast die Hälfte aller untersuchten Projekte besonders lange Fließstrecken (das sind im Epi- und Metarhithral $>5 \mathrm{~km}$, im Hyporhithral > $25 \mathrm{~km}$ und im Potamal $>50 \mathrm{~km}$ ), die noch nicht durch Stau, Restwasser, oder Wanderhindernisse unterbrochen sind.

44 der 102 KWPs würden im Falle ihrer Umsetzung morphologisch weitgehend unbeeinflusste Strecken betreffen, deren „Natürlichkeit bezüglich der Hydromorphologie“ gemäß ÖWK als hochwertig einzustufen ist. Durch dieses Sub-Kriterium fallen bei SZ 1 und 2 („Strenger Schutz“ und „WWF Energiewende") 44 KWPs in die Kategorie Ausschluss.

Der gute ökologische Zustand wird nur in SZ 1 als Ausschluss gewertet und betrifft dort 39 KWPs.

Immerhin 18 KWPs betreffen Strecken mit einem sehr guten ökologischen Zustand und 14 Projekte waren im Bereich WWRL-relevanter Natura-
2000-Gebiete geplant ${ }^{8}$. Aufgrund ihrer rechtlichen und naturschutzfachlichen Relevanz wurden diese beiden SubKriterien in den Szenarien 1 bis 3 als Ausschluss definiert (Anm.: in SZ 4 „Minimaler Schutz" gibt es keine Ausschluss-Kriterien).

Ergebnisse der ökologischen Bewertung: Von den 102 untersuchten KWPs befanden sich je 27 in Tirol und Kärnten, 13 in der Steiermark, 12 in Vorarlberg, 11 in Oberösterreich, acht in Salzburg und vier in Niederösterreich. Nach der Indexberechnung zeigte sich, dass insbesondere in den Szenarien SZ 1 bis SZ 3 ein sehr hohes Konfliktpotenzial der KWPs mit gewässerökologischen bzw. naturschutzfachlichen Aspekten besteht. So stehen bundesweit gesehen in SZ 1 rund $64 \%$ aller Projekte mit Ausschluss-Kriterien in Konflikt. Im SZ 3 sind es noch $33 \%$. Für SZ 4 hingegen ist ein geringes Konfliktpotenzial ausgewiesen. Dies ist damit zu erklären, dass hier davon ausgegangen wird, dass künftig naturschutzfachliche Aspekte nur sehr untergeordnet berücksichtigt werden. Rund $43 \%$ aller KWPs liegen hier an Strecken, deren ökologische Wertigkeit als "gering“ gewertet wurde.

Bezogen auf die Bundesländer zeigt sich insbesondere in den Szenarien „Strenger Schutz" und „WWF-Energiewende" ein hohes Konfliktpotenzial in Kärnten und der Steiermark, gefolgt von Niederösterreich und Vorarlberg. In Szenario 3 („Moderater Schutz“) fällt in Niederösterreich für 2 KWPs das Ausschluss-Kriterium („Hydromorphologischer Zustand“) der beiden anderen Szenarien weg. Jedoch sind zwei der vier Projekte weiterhin als „sehr hoch“ bzw. „hoch“ eingestuft (Abb. 8).

Ergebnisse der integrativen Bewertung: Von den 102 im Rahmen von Hy:Con untersuchen KWPs wurden im Zuge der energiewirtschaftlichen Bewertung nur 54 als zumindest „mittel“ attraktiv bewertet ( $>2,5$ Punkte). Be-

${ }^{8}$ Hierbei muss angemerkt werden, dass für die meisten dieser KWPs der exakte Wirkungsbereich zum Zeitpunkt der Berechnungen nicht bekannt war (im Zusammenhang mit dem sehr guten ökologischen Zustand waren dies 14 und bei WWRL-relevanten Natura-2000-Gebieten waren es 11 KWPs). Bei Vorliegen aktuellerer und detaillierterer Projektdaten könnten sich also bezüglich der ökologischen Bewertung noch gewisse Änderungen ergeben. 


\section{Originalarbeit}

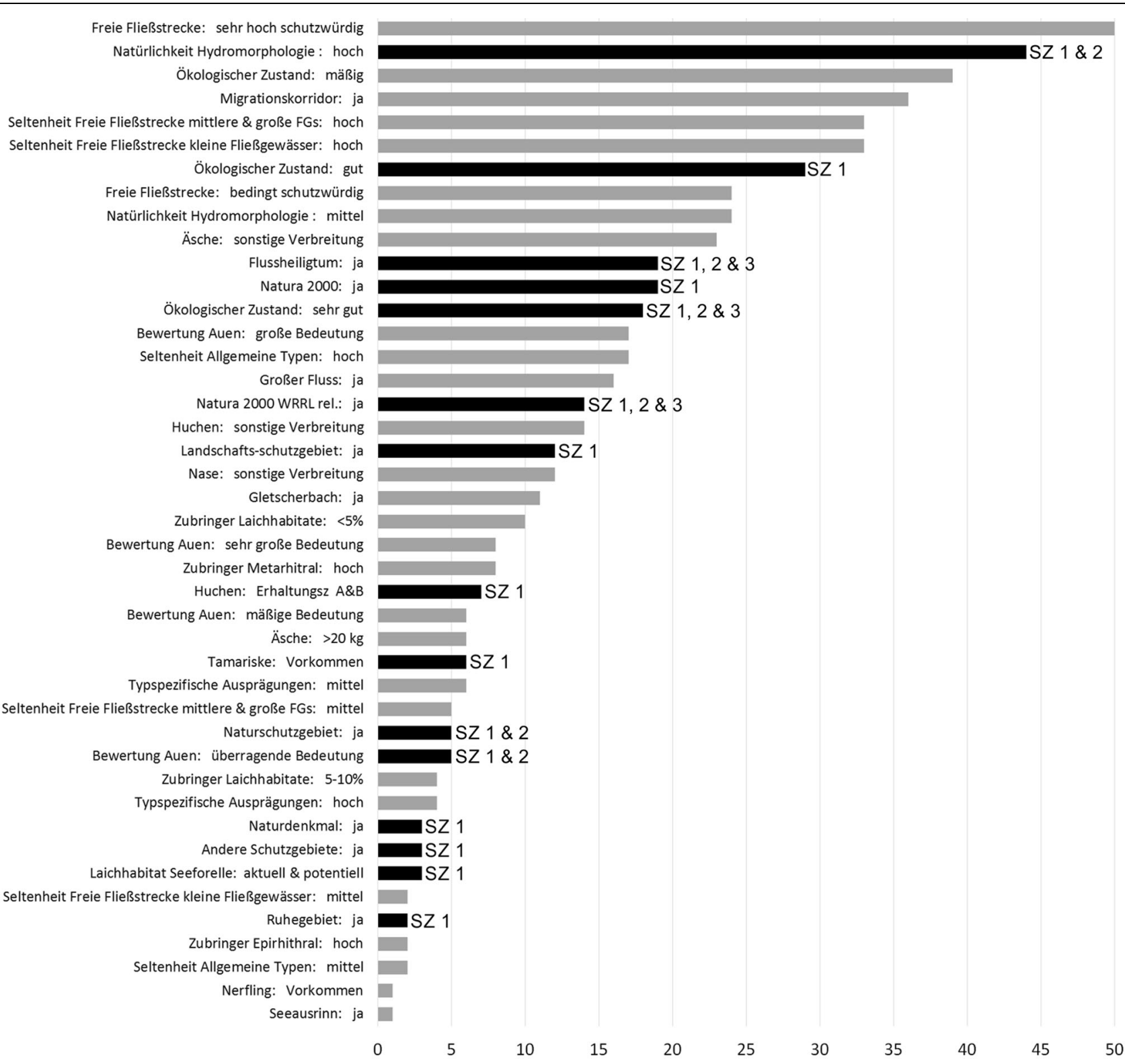

Abb. 7 Bewertungsrelevante Subkriterien des Hy:Con Modells: Anzahl der Kraftwerksprojekte für die sie zutreffend sind; Kriterien, die in mindestens einem Schutzszenario als Ausschluss gewertet wurden, sind hervorgehoben (schwarz; daneben Angabe der Szenarien in denen sie als Ausschlusskriterium eingestuft sind)

trachtet man nur die energiewirtschaftlich attraktiven KWPs, so sinkt die Zahl der „Ausschluss“-Projekte in den Szenarien 1 bis 3 jeweils ebenfalls rund um die Hälfte (Tab. 1). Im Sinne der integrativen Betrachtung sind in einem ersten Schritt insbesondere jene Projekte näher zu prüfen und ggf. zu bevorzugen, die aus energiewirtschaftlicher Sicht entsprechend hoch bewertet wurden und gleichzeitig nur geringes oder mittleres Konfliktpotenzial aufweisen (SZ 1: 16 KWPs, SZ 2: 23 KWPs; SZ 3: 32 KWPs, SZ 4: 42 KWPs). Vor dem Hintergrund, dass Kleinst- und Kleinkraftwerke nur rund $3 \%$ zur Gesamtleistung und zu rund $19 \%$ zum RAV der 102 untersuchten Projekte beitragen und vom energiewirtschaftlichen Standpunkt aus tendenziell schlechter bewertet wurden (vgl. WWF Österreich 2014; Seliger et al. 2015), sollten gerade diese Projekte aus energiepolitischer Sicht kritisch hinterfragt werden.
2.3 Fallbeispiel 3:

"Gewässerschutzplan Unser Inn" ein einzugsgebietsbezogener Planungsansatz zur Schutzpriorisierung sensibler Gewässerstrecken unter Berücksichtigung energiewirtschaftlicher Aspekte

Das letzte und aktuellste der hier vorgestellten Fallbeispiele ist der „Gewässerschutzplan Unser Inn“ (GSPI). Er wurde im Dezember 2015 im Sinne von $\S 53$ WRG als Wasserwirtschaftlicher Rahmenplan für das Einzugsgebiet des 
Szenario 1

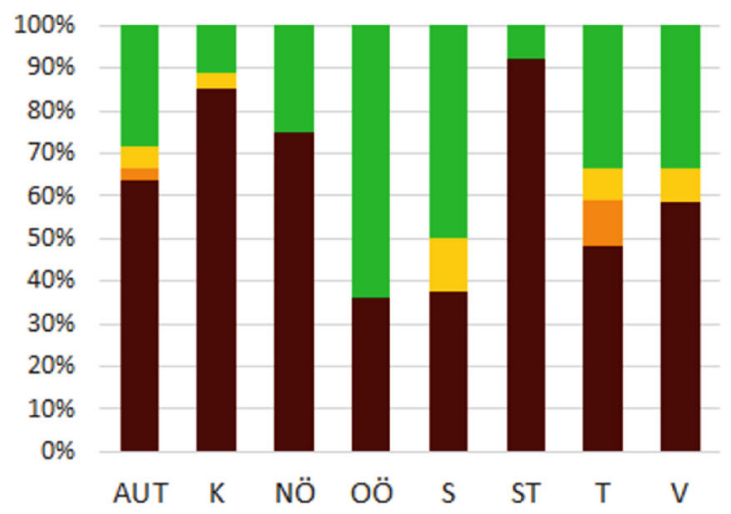

Szenario 3

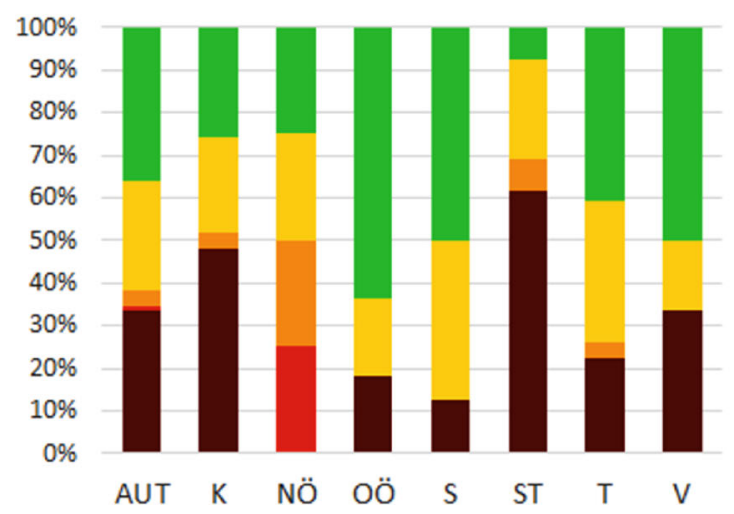

Szenario 2

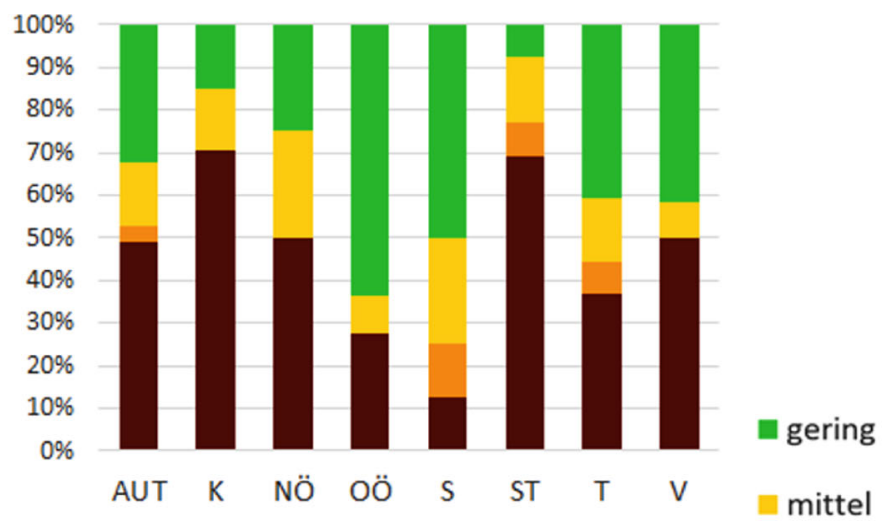

Szenario 4

noch

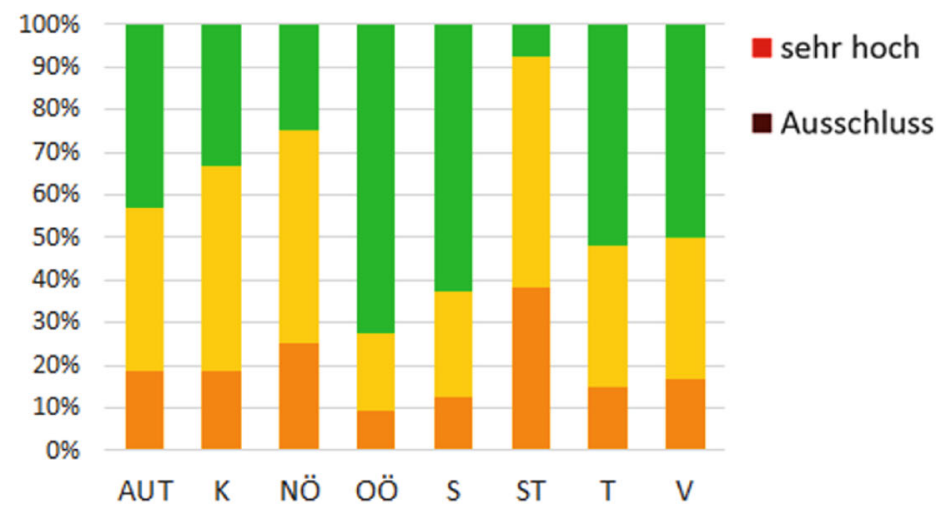

Abb. 8 Anteil der KWPs in den Bewertungsklassen für die ökologische Gesamtbewertung in Österreich (AUT) und den Bundesländern

Tiroler Inn beim Bundesministerium für Land- und Forstwirtschaft, Umwelt und Wasserwirtschaft eingereicht und stand zum Zeitpunkt des Verfassens dieses Artikels noch unter Begutachtung.

Dem GSPI liegt die Studie „Ausweisung, Bewertung und Bilanzierung der ökologischen Sensibilität von Fließgewässerstrecken" (Scheikl et al. 2015) zugrunde. Ziel der Studie war die Priorisierung aller im Untersuchungsgebiet liegenden Fließgewässer mit einem EZG $\geq 10 \mathrm{~km}^{2}$ hinsichtlich ihrer ökologischen Sensibilität und Schutzwürdigkeit. In einem weiteren Schritt galt es, diese Ergebnisse mit aktuellen Kraftwerksplanungen sowie auch dem energiewirtschaftlichen Potenzial im EZG Tiroler Inn (nach ILF Beratende Ingenieure 2011) zu überlagern. Dabei wurde der fachlich-methodische Zugang der beiden Vorgängerstudien (s. Fallbeispiele 1 und 2) weitergeführt und adaptiert. Damit liegt nun ein Preplanning-Instrument vor, das schon im Vorfeld von Kraftwerksplanungen erlaubt, die gewässerökologische und naturschutzfachliche Bedeutung von Fließgewässern bzw. Subeinzugsgebieten in einem größeren EZG strategisch zu berücksichtigen - ein Aspekt, der insbesondere bei der einzugsgebietsbezogenen energie- und wasserwirtschaftlichen Planung hohe Bedeutung hat. Im Folgenden werden Methodik und Ergebnisse der Schutzpriorisierung zusammengefasst

\subsubsection{Methodischer Zugang}

Die Vorgehensweise bei der Einstufung der Fließgewässerstrecken basierte weitestgehend auf den Methoden der Studie zum ÖMP II und ÖMP III.

Wie auch beim ÖMP II war es Ziel, das gesamte Gewässernetz in Bezug auf die Schutzwürdigkeit der einzelnen Gewässerstrecken zu untersuchen (wenn auch auf EZG- und nicht mehr auf nationaler Ebene). Hierfür sollten die umfassenden ökologischen Kriterien von Hy:Con herangezogen werden. Aus diesem Grund konnte auch bei die- ser Studie das Stichprinzip nicht mehr angewandt werden. Um die breite $\mathrm{Pa}$ lette der Kriterien zu berücksichtigen, wurde deshalb die Index-Methode dahingehend adaptiert, dass sie auf das Berichtsgewässernetz umgelegt werden konnte.

Die Bewertung erfolgte auf den ,Teilabschnitten“ des Berichtsgewässernetzes. Sie besitzen in der Regel einheitliche Längen von $500 \mathrm{~m}$ - im Gegensatz zu den Wasserkörpern, die im EZG des Tiroler Inn von $300 \mathrm{~m}$ bis über $15 \mathrm{~km}$ schwanken.

Für die Beurteilung der ökologischen Sensibilität dieser Abschnitte wurden weitestgehend dieselben Kriterien und Basiseinstufungen (Subkriterien) herangezogen, die schon bei Hy:Con verwendet wurden. Nur Kriterien, die im EZG des Tiroler Inn nicht zutreffend waren (wie z. B. Laichhabitate Seeforelle, Dohlenkrebs, Flussperlmuschel, RAMSAR-Gebiet u. a.) oder für die keine Daten verfügbar waren (Karausche und Nerfling), wurden nicht berücksichtigt. Hingegen wurden zwei neue Kriteri- 
Tab. 1 Anzahl der KWPs je ökologischer Bewertung unter Berücksichtigung der KPWs mit einer mindestens „, mittleren energiewirtschaftlichen Attraktivität“ (E > 2,5 Punkte); fett: Anzahl der KWPs mit niedrigem Konfliktpotenzial und gleichzeitig höherer energiewirtschaftlicher Attraktivität

\begin{tabular}{|c|c|c|c|c|c|c|c|c|}
\hline \multirow[t]{2}{*}{ Ökologische Wertigkeit } & \multicolumn{2}{|l|}{ SZ 1} & \multicolumn{2}{|l|}{ SZ 2} & \multicolumn{2}{|l|}{ SZ 3} & \multicolumn{2}{|l|}{ SZ 4} \\
\hline & Alle & $E>2,5$ & Alle & $E>2,5$ & Alle & $E>2,5$ & Alle & $E>2,5$ \\
\hline Ausschluss & 65 & 35 & 50 & 27 & 34 & 19 & 0 & 0 \\
\hline Sehr hoch & 0 & 0 & 0 & 0 & 1 & 0 & 0 & 0 \\
\hline Hoch & 3 & 3 & 4 & 4 & 4 & 3 & 19 & 12 \\
\hline Mittel & 5 & 2 & 15 & 9 & 26 & 18 & 39 & 24 \\
\hline Gering & 29 & 14 & 33 & 14 & 37 & 14 & 44 & 18 \\
\hline
\end{tabular}

en ergänzt, die nun in die Bewertung einflossen: In der Gruppe „Hydromorphologischer Zustand“ war dies das Kriterium „Morphologie im prioritären Sanierungsraum“ des NGP 2009 und 2015 (Entwurf, Stand Dez. 2014). Außerdem wurde in der Gruppe „rechtlich gesicherte Schutzgebiete" das Kriterium "Schutz nach Wasserrechtsgesetz“ aufgrund der Aktualität ergänzt: Im Jahr 2014 wurden im Anhang II des BGBl II Nr. 274/14 ${ }^{9}$ genau definierte Gewässerstrecken im Tiroler Oberland ausgewiesen, für die aufgrund ihres sehr guten Zustands bzw. ihrer Sensibilität ein umfassender Schutz vorgesehen ist.

Schlussendlich wurden so insgesamt 28 Einzelkriterien identifiziert, die in dieselben Gruppen wie schon bei Hy:Con zusammengefasst wurden.

Geringfügige Adaptionen z. B. hinsichtlich der Berücksichtigung von Datenlücken, erfolgten auch im Bewertungsmodell und der Indexberechnung.

Eine weitere Neuerung, die sich dadurch ergab, dass nun statt einzelner Kraftwerksstandorte das gesamte zusammenhängende Gewässernetz untersucht wurde, war die Ausweisung der „Schutzwürdigkeit“ nach der Berechnung der „Sensibilität“: Bei der Beurteilung der Sensibilität waren vielfach längere zusammenhängende Ausschluss-Strecken durch kurze Strecken unterbrochen, die niedriger bewertet wurden. Im Sinne einer vorausschauenden Planung sollten jedoch auch kurze Abschnitte möglichst von größeren Eingriffen ausgenommen werden, da dadurch auch die direkt angrenzenden Ausschluss-Strecken betroffen sein können. Je nach Einzugsgebietsgröße der Teilabschnitte wur-

\footnotetext{
9 „Verordnung des BMLFUW vom 3. November 2014 über die Anerkennung der im Rahmenplan Tiroler Oberland dargestellten wasserwirtschaftlichen Ordnung als öffentliches Interesse“.
}

den bestimmte Höchstlängen dieser Zwischenstücke definiert, bis zu denen sie zum Ausschluss ergänzt werden konnten $\left(<10 \mathrm{~km}^{2}: 1 \mathrm{~km}\right.$; 10 bis $<100 \mathrm{~km}^{2}: 2 \mathrm{~km} ; 100$ bis $<1.000 \mathrm{~km}^{2}$ : $5 \mathrm{~km} ;>1.000 \mathrm{~km}^{2}: 10 \mathrm{~km}$ ).

Aufgrund der praktischen Ausrichtung des Bewertungsmodells erfolgten die Endbewertung und die Einstufung der Subkriterien nicht mehr szenarienbasiert. Als Grundlage für die Einstufung der Subkriterien hinsichtlich ihrer ökologischen Wertigkeit wurde das Szenario 2 aus Hy:Con herangezogen.

\subsubsection{Ausgewählte Ergebnisse}

Von den insgesamt rund $2.400 \mathrm{~km}$ Fließgewässerstrecken, die im Rahmen der GSPI-Studie untersucht wurden, waren rund $46 \%$ als besonders schützenswerte Strecken auszuweisen und damit der Kategorie „Ausschluss - bezogen auf den Wasserkraftausbau“ zuzuordnen. Abgesehen von der langen Fließstrecke des Inn zwischen Innsbruck und Kirchbichl waren dies vor allem weitgehend unbeeinflusste Oberläufe. Teilabschnitte mit einem EZG $<100 \mathrm{~km}^{2}$ hatten einen besonders hohen Anteil an Ausschluss-Strecken. Oberläufe mit einem EZG von weniger als $10 \mathrm{~km}^{2}$ hatten sogar einen Anteil von beinahe $70 \%$ an Ausschluss-Strecken (vgl. Abb. 9 und 10). Fast $80 \%$ aller Ausschluss-Strecken basierten, zumindest zum Teil, auf dem Kriterium „Natürlichkeit Hydromorphologie ${ }^{\text {"10 }}$; auf rund $60 \%$ aller Ausschluss-Strecken war der ökologische Zustand "sehr gut“. Schutzgebiete nahmen bei der Gesamtbetrachtung großteils eine untergeordnete Rolle ein (zwischen 0,6\% und $5,5 \%)$. Nur das Kriterium „Schutz nach Wasserrechtsgesetz" traf auf immerhin $44 \%$ aller Ausschluss-Strecken zu.

${ }^{10}$ Entspricht „Hydromorphologischer Zustand“ im ÖMP III.
Eine niedrigere Priorität bzgl. der Schutzwürdigkeit wurde vor allem an Mittel- und Unterläufen der Inn-Zubringer bzw. an Strecken mit bereits vorhandener energiewirtschaftlicher Nutzung (insbesondere Restwasser) ausgewiesen (25\% des Gewässernetzes gering und $21 \%$ mittel).

\section{Schlussfolgerungen}

Derzeit erfolgen wirtschaftlich-ökologische Abwägungs- und Planungsprozesse hinsichtlich des Wasserkraftausbaus überwiegend auf Bundesländerebene (z. B. über Beschlüsse der Landesregierungen oder Regionalprogramme). Im Sinne der Umsetzung bestehender, nationaler Strategien und Zielsetzungen ist es jedoch notwendig, auch großmaßstäbliche, bundesweite Analysen und Bewertungen der Ist-Situation sowie geplanter Ausbaumaßnahmen (wie beispielsweise der Wasserkraft) durchzuführen. So wurde auch seitens der EU und in internationalen Leitlinien für den Donau- und den Alpenraum empfohlen, eine fachlich-integrative Beurteilung von Gewässertrecken hinsichtlich deren Eignung für den Wasserkraftausbau auf räumlich übergeordneter Ebene durchzuführen (z. B. Plattform Wasserwirtschaft im Alpenraum 2011; ICPDR 2013).

Die hier vorgestellten Fallbeispiele zeigen, dass es möglich ist, auch auf großmaßstäblicher Ebene Grundlagen für strategische Planungsprozesse in konsistenter und transparenter Form $\mathrm{zu}$ erstellen und Entscheidungsprozesse damit zu unterstützen. Unsere Analysen basieren fast ausschließlich auf öffentlich verfügbaren Daten, die regelmäßig aktualisiert werden und orientieren sich an den aktuellen rechtlichen Rahmenbedingungen. Keines der Fallbeispiele hat den Anspruch, alle lokalen Gegebenheiten abzudecken und Einzelfallprüfungen $\mathrm{zu}$ ersetzen. Primäres Ziel war es, nationale bzw. 


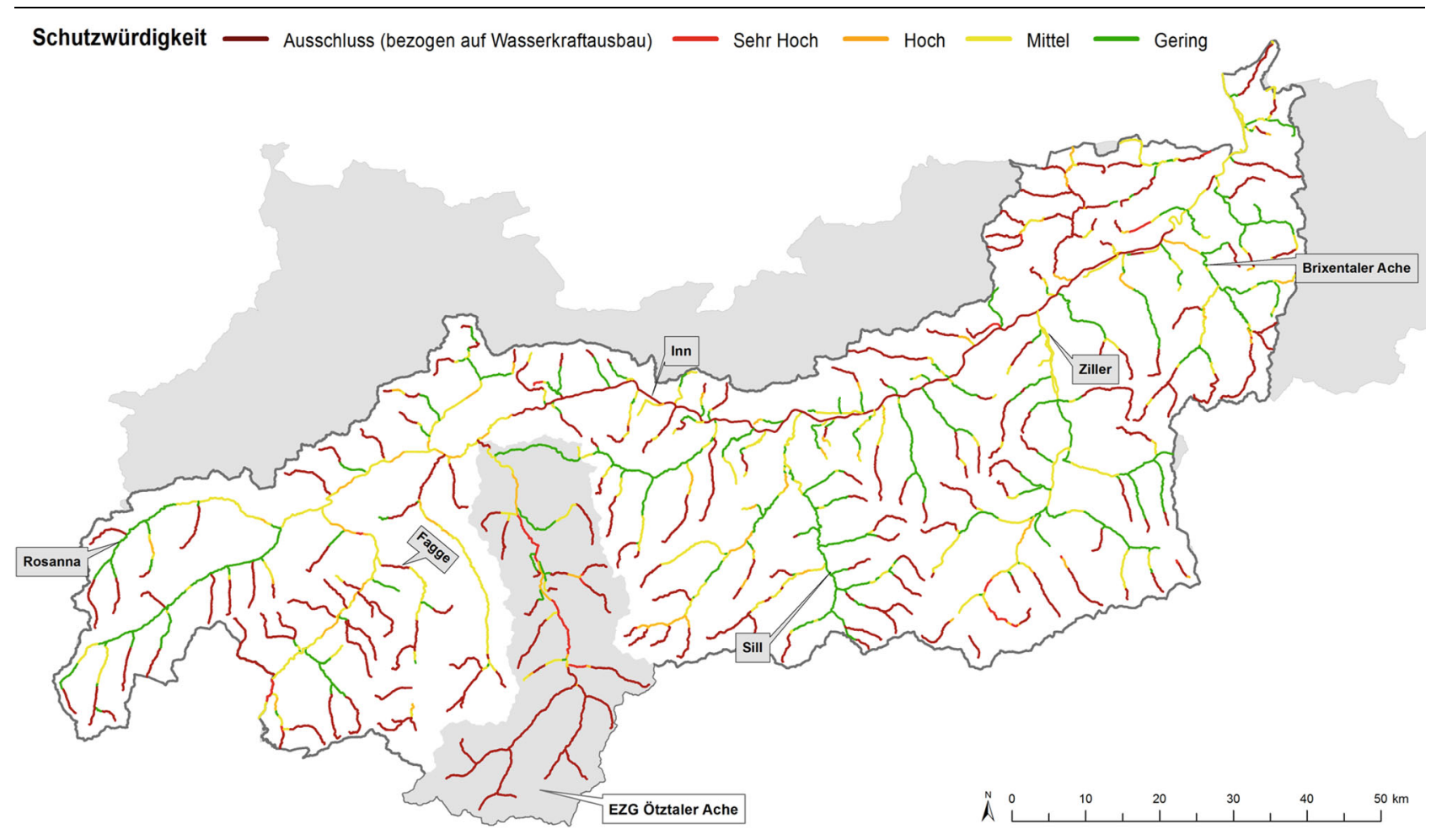

Abb. 9 RäumlicheDarstellung derSchutzwürdigkeitvonFließgewässerstrecken(EZG $\left.\geq 100 \mathrm{~km}^{2}\right)$ imEZG des Tiroler InngemäßGSPI (WWF Österreich 2015)

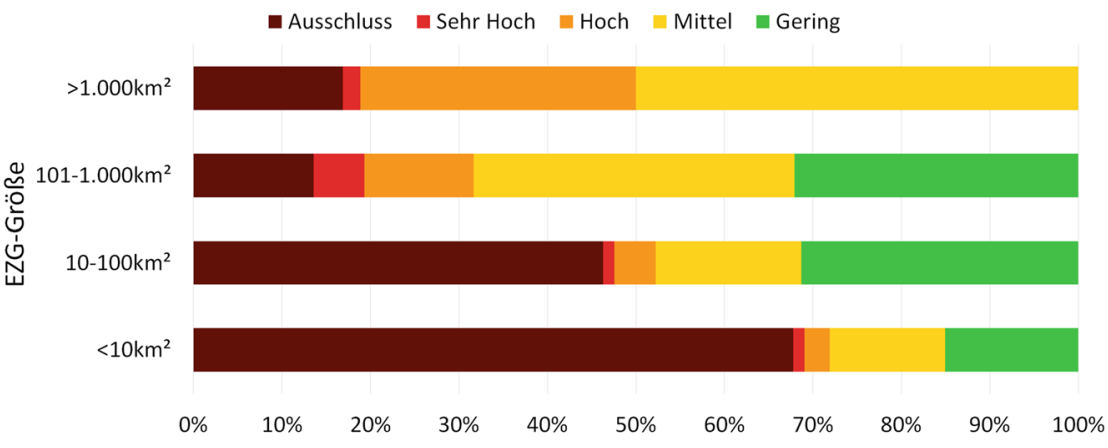

Abb. 10 Verteilung der Ausschluss-Strecken auf die EZG-Größen der Teilabschnitte

(über-)regionale Planungsprozesse mittels fundierter Fachgrundlagen in ihrer ersten Phase der Entscheidungsfindung zu unterstützen. Dabei können insbesondere die Studien zum ÖMP II und Hy:Con als wesentliche Entscheidungshilfen dienen. Der GSPI hat, konzipiert als Wasserwirtschaftlicher Rahmenplan, neben dieser Funktion auch das Ziel, rechtlich verbindliche Festlegungen zu vorzuschlagen.

Ein wesentlicher Vorteil der dargestellten Modelle ist ihre räumliche und thematische Übertragbarkeit und damit auch ihre Flexibilität. So können die Kriterien und deren Gewichtung bzw. Einzelbewertung an geänderte rechtliche Vorgaben angepasst werden.
Auch die Kriterienliste selbst kann je nach Raumbezug adaptiert werden (wie auch die Hy:Con-Kriterien im Rahmen des GSPI an die Gegebenheiten des EZG des Tiroler Inn angepasst wurden). Sie kann außerdem dem Stand der Forschung sowie der Datenaktualisierung und -verfügbarkeit angepasst werden und aktualisiert in die Modelle einfließen. So könnten (1) mögliche Kumulationseffekte von energiewirtschaftlichen Nutzungen in die Bewertung integriert werden, (2) die Auswahl von Schlüsselarten, Schutzgebietskategorien etc. aktualisiert oder (3) die methodischen Ansätze durch zusätzliche sozio-ökonomische oder -ökologische Indikator-
Gruppen ergänzt werden - um hier nur einzelne Beispiele zu nennen.

Außerdem gilt es, neben einer umfassenden fachlichen Bewertung auch die politische und gesellschaftliche Akzeptanz und damit auch die Anwendung solcher strategischer Planungen und deren Umsetzung zu fördern. Die Integration partizipativer Methoden in derartigen Planungs- und Entscheidungsprozesse ist ein geeignetes sowie rechtlich auch verankertes Instrument (vgl. Art. 14 WRRL) und wird aktuell im Rahmen des Interreg-AlpineSpace-Projekts SPARE ${ }^{11}$ im Kontext der Harmonisierung von Schutz- und Nutzungsansprüchen an alpine Fließgewässer auf lokaler bis hin zu supraregionaler Ebene erprobt und evaluiert.

\section{Danksagung}

Die hier vorgestellten Fallbeispiele wurden durch den WWF Österreich finanziert. Besonderer Dank für die $\mathrm{Zu}$ sammenarbeit ergeht an Christoph Litschauer, Gebhard Tschavoll und Bettina Urbanek. Die energiewirtschaftlichen Analysen und Bewertungen wurden von

11 „Strategic Planning for Alpine River Ecosystems“, www.alpine-space.eu/SPARE. 
Jürgen Neubarth (e3 consult $\mathrm{GmbH}$ ) durchgeführt (ÖMP III) bzw. fachlich begleitet (GSPI). Außerdem danken wir für die Datenbereitstellung dem BMLFUW, dem Umweltbundesamt, den Ämtern der Landesregierungen, insbesondere den Ländern Niederösterreich und Tirol für die direkte Unterstützung bzgl. Datenrecherche und -übermittlung. Georg Reitzner und Johannes Rein (ILF Beratende Ingenieure) sowie
Helmut Kudrnovski, Thomas Ofenböck und Jürgen Petutschnig danken wir für Daten und Fachinformationen.

Open access funding provided by University of Natural Resources and Life Sciences Vienna (BOKU)

Open Access Dieser Artikel wird unter der Creative Commons Namensnennung 4.0 International Lizenz (http:// creativecommons.org/licenses/by/4. 0/deed.de) veröffentlicht, welche die Nutzung, Vervielfältigung, Bearbeitung, Verbreitung und Wiedergabe in jeglichem Medium und Format erlaubt, sofern Sie den/die ursprünglichen $\mathrm{Au}$ tor(en) und die Quelle ordnungsgemäß nennen, einen Link zur Creative Commons Lizenz beifügen und angeben, ob Änderungen vorgenommen wurden.
Amt der Oberösterreichischen Landesregierung (2015): Publikationsbericht: Oö. Wasserkraftpotentialanalyse 2012/13. Abschätzung und Evaluierung des energetischen Revitalisierungs- und Ausbaupotentials an umweltgerechten Standorten an mittleren und größeren Gewässern in Oberösterreich.

Amt der Tiroler Landesregierung (2011): Wasserkraft in Tirol: Kriterienkatalog. Kriterien fü eine nachhaltige Nutzung der Wasserkraft in Tirol. Innsbruck

Amt der Vorarlberger Landesregierung (2011) Schritt für Schritt zur Energieautonomie in Vorarlberg. 101 enkeltaugliche Maßnahmen. Maßnahmenplan bis 2020 - Schlussbericht. Bregenz BMFL, BMUJF \& WWF (1998): Das Buch der Flüsse - 74 Flußstrecken von österreichweite Bedeutung. Wien

BMLFUW (2010): Nationaler Gewässerbewirtschaftungsplan 2009 - NGP 2009 (BMLFUWUW.4.1.2/0011-I/4/2010).

BMLFUW (2012): Österreichischer Wasserkatalog Wasser schützen - Wasser nutzen; Kriterien zur Beurteilung einer nachhaltigen Wasserkraftnutzung-Erlass.

BMLFUW (2015): Nationaler Gewässerbewirtschaftungsplan 2015 (Entwurf).

BMWFJ \& BMLFUW (2010): EnergieStrategie Österreich - Maßnahmenvorschläge. Wien CIS (2006): WFD and Hydro-morphological pressures. Policy Paper. Focus on hydropower, navigation and flood defence activities Recommendations for better policy integration. Energie-Control Austria (2015a): Bestandsstatistik - Kraftwerkspark

Engpassleistung nach Kraftwerkstypen zum 31. Dezember 2014. http://www.e-control. at/documents/20903/503039/BeStGes-2014 KW2EPLTyp.xlsx/31282308-5ee6-465c-a8e5fbc4a3429d58. Gesehen 29.04.2016

Energie-Control Austria (2015b): Betriebsstatistik - Jahresreihen. Gesamte Elektrizitätsversorgung: Jährliche Erzeugung elektrischer Energie. http// www.e-control.at/documents/ 20903/447897/BStGes-JR4_Erzgg.xlsx/1fff1065f875-4510-bfb2-aafa9d4e8582?version=1.1. Gesehen 29.04.2016
Europäische Kommission (2012): Bericht der Kommission an das Europäische Parlament und den Rat über die Umsetzung der Wasserrahmenrichtlinie (2000/60/EG) Bewirtschaftungsplän für Flusseinzugsgebiete. COM(2012) 670 final. Brüssel

Europäische Kommission (2015): Wasserrahmenrichtlinie und Hochwasserrichtlinie - Maßnahmen zum Erreichen eines guten Gewässerzustands in der EU und zur Verringerung der Hochwasserrisiken.Mitteilung der Kommission an das Europäische Parlament und den Rat. COM(2015) 120 final. Brüsse

Habersack, H., Wagner, B., Hauer, C. et al (2011): DSS_KLIM:EN: Entwicklung eines Decision Support Systems zur Beurteilung des Wechselwirkungen zwischen Klimawandel, Energie aus Wasserkraft und Ökologie. Endbericht. Wien ICPDR (2013): Leitlinien zum nachhaltigen Ausbau der Wasserkraft im Einzugsgebiet de Donau. Internationale Kommission zum Schutz der Donau, Wien

ILF Beratende Ingenieure (2011): Wasserkraft in Tirol: Potentialstudie. Ermittlung des noch verfügbaren Wasserkraftpotenzials in Tirol.

Lazowski, W., Schwarz, U., Essl, F. et al (2011): Aueninventar Österreich -Bericht zur bundesweiten Übersicht der Auenobjekte. Anhang: Karte, Datenbank. BMLFUW \& UBA

Muhar, S., Schmutz, S., Seliger, C. et al (2013) Kraftwerksdatenbank des Projekts „Anwendun und Analyse ökologischer Kriterien zur Priorisierung von Schutzmaßnahmen an österreichischen Fließgewässern, Fallstudie.“

OECD \& IEA (2014): IEA data from World Energy Balances online data service 2014 edition. www. iea.org/statistics. (Datenbankabfrage unter http://databank.worldbank.org am 13.03.2016) Petutschnig, J. (2009): Rote Liste der Flusskrebse (Decapoda) Österreichs. In: Rote Liste gefährdeter Tiere Österreichs. Grüne Reihe Band 14/3 (Flußkrebse, Köcherfliegen, Skorpione, Weberknechte, Zikaden). BMLFUW

Plattform Wasserwirtschaft im Alpenraum (2011): Gemeinsame Leitlinien für die Kleinwasserkraftnutzung im Alpenraum. Ständiges Sekretariat der Alpenkonvention, Innsbruck
PÖYRY (2008): Wasserkraftpotentialstudie Österreich. Endbericht. VEÖ

Reischüitz, A., Reischütz, P. L (2007): Rote Liste der Weichtiere (Mollusca) Österreichs. In: Rote Liste gefährdeter Tiere Österreichs; Grüne Reihe Band 14/2 (Kriechtiere, Lurche, Fische, Nahtfalter, Weichtiere). BMLFUW

Scheikl, S., Loach, A., Muhar, S. (2015): Gewässerschutzplan „Unser Inn“- Wasserwirtschaftlicher Rahmenplan für das Flusseinzugsgebiet ds Tiroler Inn. Version II. Fachbeitrag „Ausweisung, Bewertung und Bilanzierung der ökologischen Sensibilität von Fließgewässerstrecken.

Seliger, C., Scheikl, S., Schmutz, S. et al (2015): Hy:Con: A Strategic Tool For Balancing Hydropower Development And Conservation Needs. River Res Appl. doi: 10.1002/rra.2985

Wagner, B, Hauer, C, Schoder, A, Habersack, H. (2015): A review of hydropower in Austria: Past, present and future development. Renew Sustain Energy Rev 50:304-314. doi 10.1016/j.rser.2015.04.169

Wolfram, G., Mikschi, E. (2007): Rote Liste de Fische (Pisces) Österreichs. In: Rote Liste gefährdeter Tiere Österreichs. Grüne Reihe Band 14/2 (Kriechtiere, Lurche, Fische, Nachtfalter, Weichtiere). BMLFUW

WWF Österreich (2010): Ökomasterplan Stufe II - Schutz für Österreichs Flussjuwele - Zustand und Schutzwürdigkeit der Österreichischen Fließgewässer mit einem Einzugsgebiet größer $10 \mathrm{~km}^{2}$ - Ergebnisse und Handlungsempfehlungen. WWF Österreich, Wien

WWF Österreich (2014): Ökomasterplan Stufe III - Schutz für Österreichs Flussjuwele. Strategische Betrachtungen für einen ausreichenden Gewässerschutz sowie einen ökologisch verträglichen Ausbau der Wasserkraft in Österreich. WWF Österreich

WWF Österreich (2015): Gewässerschutzplan „Unser Inn“ - Wasserwirtschaftlicher Rahmenplan für das Flusseinzugsgebiet ds Tiroler Inn. Version II. 